Prevalence of sensitization and allergy to kiwi fruit (Actinidia deliciosa) among adults with allergic diseases

\title{
Prevalencia de sensibilización y alergia al kiwi (Actinidia deliciosa) en adultos con enfermedades alérgicas
}

\author{
Tonatiuh Ramses Bedolla-Pulido, ${ }^{1}$ Silvia Araceli Álvarez-Corona, ${ }^{1}$ Tonantzin Isis Bedolla-Pulido, ${ }^{2}$ \\ Benjamín Uribe-Cota, ${ }^{1}$ Tania González-Mendoza, ${ }^{2}$ Martín Bedolla-Barajas ${ }^{1}$
}

\begin{abstract}
Background: The kiwi fruit (Actinidia deliciosa) is a food that has been recognized for its allergenic capability for more than 30 years. In general, kiwi allergy is characterized by local discomfort, but systemic reactions such as rash, angioedema, rhinitis, conjunctivitis or anaphylaxis can be triggered.

Objective: To determine the prevalence of sensitization and allergy to kiwi in adults with allergic diseases.

Methods: By means of a cross-sectional, retrolective study, data corresponding to 370 patients aged $\geq 16$ years were analyzed.

Results: 226 patients had positive skin reaction against aeroallergens. The prevalence of food sensitization was $84 / 226(37.2 \% ; 95 \% \mathrm{Cl}=31.1$ to 43.6$)$. Overall, the prevalence of sensitization to kiwi fruit was $15 / 226(6.6 \% ; 95 \% \mathrm{Cl}=3.9$ to 10.7$)$, and of kiwi allergy, $2 / 15(13.3 \% ; 95 \% \mathrm{Cl}$ $=2.5$ to 39.1); one patient had symptoms consistent with oral allergy syndrome, and another, gastrointestinal and cutaneous manifestations.

Conclusion: The prevalence of sensitization to kiwi fruit is not a rare event; in contrast, symptoms related to its consumption are uncommon.
\end{abstract}

Keywords: Food hypersensitivity; Actinidia; Actinidiaceae; Allergic rhinitis; Asthma

Este artículo debe citarse como: Bedolla-Pulido TR, Álvarez-Corona SA, Bedolla-Pulido TI, Uribe-Cota B, González-Mendoza T, Bedolla-Barajas M. Prevalencia de sensibilización y alergia al kiwi (Actinidia deliciosa) en adultos con enfermedades alérgicas. Rev Alerg Mex. 2018;65(1):19-24

ORCID

Tonatiuh Ramses Bedolla-Pulido, 0000-0002-5292-0728; Silvia Araceli Álvarez-Corona, 0000-0001-9828-1397; Tonantzin Isis Bedolla-Pulido, 0000-0002-3374-8061; Benjamín Uribe-Cota, 0000-0002-5946-960X; Tania González-Mendoza, 0000-0003-4121-0293; Martín Bedolla-Barajas, 0000-0003-4915-1582

${ }^{1}$ Hospital Civil de Guadalajara Dr. Juan I. Menchaca, Servicio de Alergia e Inmunología Clínica, Guadalajara, Jalisco, México. ${ }^{2}$ Universidad de Guadalajara, Centro Universitario de Ciencias de la Salud, Guadalajara, Jalisco, México.

\author{
Correspondencia: Martín Bedolla-Barajas \\ drmbedbar@gmail.com \\ Recibido: 2017-07-08 \\ Aceptado: 2017-09-12 \\ DOI: $10.29262 /$ ram.v65i1.293
}




\section{Resumen}

Antecedentes: El kiwi (Actinidia deliciosa) es un alimento reconocido por su capacidad alergénica desde hace más de 30 años. Por lo general, la alergia al kiwi se caracteriza por molestias locales, pero pueden desencadenarse reacciones sistémicas como urticaria, angioedema, rinitis, conjuntivitis o anafilaxia.

Objetivo: Determinar la prevalencia de sensibilización y alergia al kiwi en adultos con enfermedades alérgicas.

Métodos: Mediante un estudio transversal se analizaron los datos correspondientes a 370 pacientes $\geq 16$ años de edad.

Resultados: Tuvieron reacción cutánea positiva contra aeroalérgenos 226/370 pacientes. La prevalencia de sensibilización a alimentos fue 84/226 (37.2 \%; IC $95 \%=31.1$ a 43.6). En general, la prevalencia de sensibilización al kiwi fue de 15/226 (6.6 \%; IC $95 \%=3.9$ a 10.7) y de alergia al kiwi de 2/226 (0.9 \%; IC $95 \%=0.03$ a 3.4), un paciente tuvo síntomas compatibles con síndrome de la alergia oral y otro, manifestaciones gastrointestinales y cutáneas.

Conclusión: La prevalencia de sensibilización al kiwi no es un evento raro; en contraste, los síntomas relacionados con el consumo del fruto son poco frecuentes.

Palabras clave: Hipersensibilidad a alimentos; Actinidia; Actinidiaceae; Rinitis alérgica; Asma

\section{Antecedentes}

La planta del kiwi (Actinidia deliciosa) es originaria de China; su fruto se comercializa mundialmente desde 1970. El fruto del kiwi es una baya ovalada de piel marrón y cubierta de pelos finos, que puede llegar a medir hasta $8 \mathrm{~cm}$ de largo; la pulpa del fruto es verde brillante y contiene numerosas semillas negras. ${ }^{1}$ En promedio, un kiwi pesa $74 \mathrm{~g}$ y su aporte calórico es de $45 \mathrm{kcal}$, generado a partir de su contenido de proteínas, lípidos y carbohidratos. ${ }^{2}$

El kiwi es un alimento reconocido por su capacidad alergénica desde hace más de 30 años. Por lo general, la alergia al kiwi puede provocar desde molestias locales, como el síndrome de alergia oral y la urticaria por contacto, hasta molestias sistémicas, como urticaria, angioedema, rinitis, conjuntivitis o anafilaxia. $^{3}$

Se ha identificado que el kiwi contiene al menos 31 alérgenos, ${ }^{4}$ sin embargo, cinco de ellos son los mayormente implicados en reacciones alérgicas: quitinasa (Act d 3), actidina (Act d 1), kiwelina (Act d 2), TLP (Act d 4) y cistatina (Act d 4). ${ }^{3}$

Dado que los estudios que abordan la alergia al kiwi en población adulta son escasos en México, esta investigación tuvo como objetivos determinar la prevalencia de sensibilización y alergia al kiwi, así como describir las características clínicas en este tipo de pacientes.

\section{Métodos}

Los datos fueron obtenidos de forma retrolectiva, mediante la aplicación de una cédula estructurada que se requisitó a partir de los expedientes clínicos de los pacientes que acudieron por primera vez a un servicio de alergología y que requirieron pruebas cutáneas; el periodo de revisión comprendió de enero a diciembre de 2016. Se incluyó la información correspondiente a los pacientes con edad $\geq 16$ años, con historia de enfermedad alérgica, asma, rinitis alérgica, dermatitis atópica o alergia a alimentos.

De manera convencional, en nuestro servicio se realizan pruebas cutáneas a alimentos, pero solo en los pacientes que muestran reactividad cutánea positiva al menos a uno de los aeroalérgenos probados. En ese mismo momento o en la próxima visita médica, se interrogó por la presencia de síntomas producidos por el alimento sensibilizante.

Sensibilización al fruto del kiwi se definió por la presencia de una prueba cutánea por punción, positiva al alérgeno de ese fruto $\left(\right.$ Allerquim $\left.^{\circledR}\right)$; para de- 
finir alergia al kiwi, además de lo anterior, durante el interrogatorio clínico, el paciente debió referir síntomas dentro de las siguientes dos horas posteriores a la ingesta del fruto.

Las pruebas cutáneas se realizaron colocando el alérgeno sobre la región volar de ambos antebrazos de cada paciente, luego, se hizo una punción con una lanceta estandarizada (Hollister-Stier ${ }^{\mathbb{R}}$ ). Después de transcurridos 15 minutos, se realizó la interpretación de las pruebas cutáneas de acuerdo con estándares internacionales; ${ }^{5}$ la prueba se consideró positiva cuando el tamaño de la pápula fue $\geq 3 \mathrm{~mm}$ en comparación con el control negativo.

\section{Análisis estadístico}

La frecuencia de sensibilización y alergia al fruto del kiwi se presenta como prevalencia; además se estimaron intervalos de confianza a $95 \%$ (IC $95 \%$ ) para proporciones. El sistema IBM SPSS (IBM Co., Armanok, NY, Estado Unidos) versión 20.0 para Windows sirvió para procesar los datos.

\section{Resultados}

En total se evaluaron los datos correspondientes a 370 pacientes, de ellos, 226 tuvieron al menos una prueba cutánea positiva a alguno de los aeroalérgenos probados; en esos mismos pacientes, la prevalencia de sensibilización a alimentos fue 84/226 (37.2\%; IC $95 \%=31.1$ a 43.6).

La sensibilización al kiwi tuvo una prevalencia de $15 / 226$ casos $(6.6 \%$; IC $95 \%=3.9$ a 10.7$) ; 13$ de esos casos correspondieron a mujeres y los otros dos a hombres; la edad mediana del grupo fue 38 años, un mínimo de 17 años y un máximo de 74 años (cuadro 1). Rinitis alérgica tuvieron 13/15 (86.7\%), asma en $6 / 15(40 \%)$ y alergia a alimentos en $2 / 15(13.3 \%)$.

\begin{tabular}{|c|c|c|c|c|c|c|c|}
\hline Caso & Sexo & $\begin{array}{c}\text { Edad } \\
\text { (años) }\end{array}$ & $\begin{array}{l}\text { Rinitis } \\
\text { alérgica }\end{array}$ & Asma & $\begin{array}{l}\text { Alergia a } \\
\text { alimentos }\end{array}$ & $\begin{array}{l}\text { Sensibilización } \\
\text { a otros alimentos }\end{array}$ & Síntomas \\
\hline 1 & $\mathrm{H}$ & 68 & - & + & - & Durazno & \\
\hline 2 & $\mathrm{H}$ & 31 & + & - & - & $\begin{array}{l}\text { Aguacate, almendra, cacahuate, cama- } \\
\text { rón, durazno, fresa, mango, manzana, } \\
\text { melón, piña, zanahoria }\end{array}$ & \\
\hline 3 & M & 50 & + & + & - & Mango & \\
\hline 4 & M & 46 & + & + & - & Mango & \\
\hline 5 & M & 38 & + & - & - & - & \\
\hline 6 & M & 47 & + & - & - & - & \\
\hline 7 & $M$ & 17 & + & + & + & Soja & $\begin{array}{l}\text { Vómito, náusea, dolor } \\
\text { y distensión abdomi- } \\
\text { nal y exantema }\end{array}$ \\
\hline 8 & $M$ & 52 & + & - & + & $\begin{array}{l}\text { Aguacate, almendra, camarón, durazno, } \\
\text { mango, melón, plátano, piña, soya }\end{array}$ & $\begin{array}{l}\text { Prurito palatino y } \\
\text { opresión faríngea }\end{array}$ \\
\hline 9 & M & 74 & - & + & - & Durazno, fresa, mango, pera, plátano & \\
\hline 10 & M & 21 & + & - & - & $\begin{array}{l}\text { Almendra, camarón, durazno, fresa, } \\
\text { melón, soja }\end{array}$ & \\
\hline 11 & M & 59 & + & - & - & $\begin{array}{l}\text { Camarón, durazno, fresa, nuez, piña, } \\
\text { soya }\end{array}$ & \\
\hline 12 & M & 32 & + & - & - & Mango, manzana, piña, soja & \\
\hline 13 & M & 35 & + & - & - & - & \\
\hline 14 & M & 33 & + & - & - & Piña & \\
\hline 15 & M & 21 & + & + & - & - & \\
\hline \multicolumn{8}{|c|}{$\mathrm{H}=$ hombre, $\mathrm{M}=$ mujer } \\
\hline
\end{tabular}


Solamente $2 / 226$ casos ( $0.9 \%$; IC $95 \%=0.03$ a 3.4 ) manifestaron síntomas después de consumir el kiwi; uno de ellos fue compatible con el síndrome de la alergia oral, el otro tuvo manifestaciones gastrointestinales y cutáneas. La monosensibilización al kiwi se observó en cuatro pacientes sensibilizados a alimentos; en dos pacientes, además del kiwi, se detectó sensibilización a 10 y 11 alimentos, respectivamente. Entre los alimentos sensibilizadores que mayormente acompañaron al kiwi estuvieron el durazno y el mango (40 \% cada uno). En ninguno de los 15 casos hubo síntomas con la exposición a productos derivados del látex.

En los pacientes sensibilizados al kiwi, los aeroalérgenos que mostraron reactividad cutánea más frecuente fueron ácaros del polvo casero (67\%), gato (67\%), Fraxinus udhei (60\%), Prosopis juliflora (53 \%), Ambrosia elatior (53\%), Chenopodium ambrosoides (47\%), Shorgum halepensis (40 \%) y Phleum pratense (40\%) (figura 1). Por su parte, en los pacientes alérgicos al kiwi, uno de ellos estuvo sensibilizado a los siguientes aeroalérgenos:

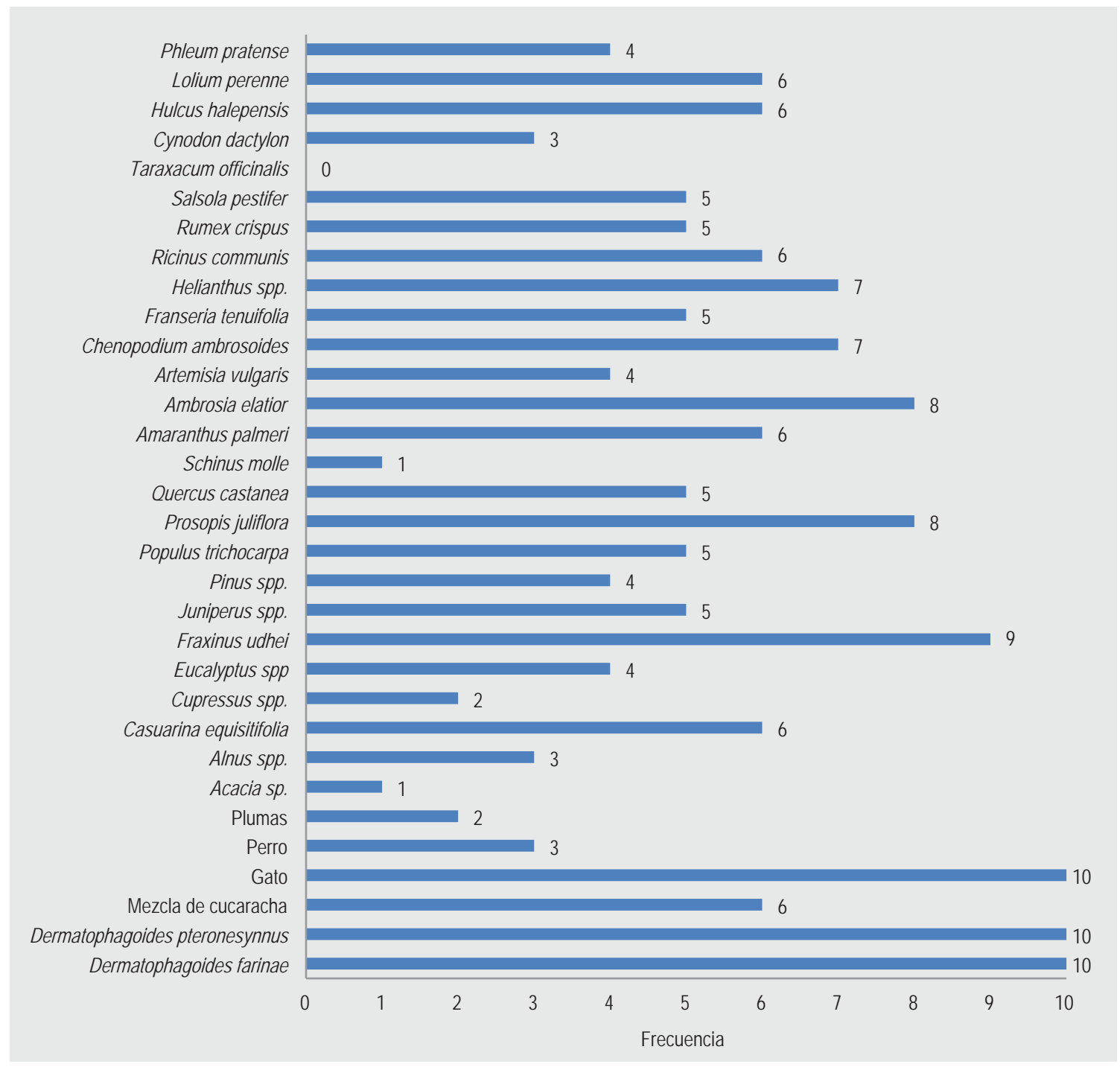

Figura 1. Frecuencia de reacción cutánea positiva a aeroalérgenos en 15 pacientes con sensibilización al kiwi. 
ácaros del polvo casero, epitelio de gato, alisos, casuarinas, cipreses, juníperos, álamos, mezquites, quelite, estafiate, epazote, amargosilla, higuerilla, lengua de vaca y diversos pastos. El otro paciente estuvo sensibilizado a epitelio de gato, casuarinas, fresnos, álamos, pinos, mezquites, robles, quelite, epazote, girasol, higuerilla, lengua de vaca y pastos.

\section{Discusión}

En población adulta con enfermedades alérgicas, la prevalencia de sensibilización al kiwi no es un evento infrecuente; en comparación, la presencia de síntomas relacionados con el consumo del fruto es un suceso raro.

En Latinoamérica, el estudio de la alergia al kiwi es limitado. Así, nuestra investigación ofrece información relevante en ese sentido. La mayoría de los análisis realizados hasta el momento han centrado su atención en la prevalencia de autorreporte de alergia y no en la frecuencia de sensibilización o alergia al kiwi; por ejemplo, en Europa la prevalencia acumulada en población adulta fue de $5.2 \%$, mucho mayor en España, donde se ubicó por arriba de $10 \%$, en cambio, en el Reino Unido, la prevalencia fue de $1.5 \%{ }^{6}$ En el occidente de México se informó que $0.5 \%$ de la población adulta joven percibió ser alérgica al kiwi; ${ }^{7}$ en cambio, hasta $1.4 \%$ de los estudiantes de una universidad pública señalaron tener síntomas después de comer kiwi. ${ }^{8}$ Sin embargo, al tratarse de estudios de percepción, se recomienda interpretar los resultados con reserva. En cuanto a la prevalencia de sensibilización al kiwi, estudios realizados en la India, Estados Unidos o Australia, han mostrado las siguientes cifras: $8.2,5.2$ y $4.1 \%$, respectivamente, ${ }^{6,9}$ y estos hallazgos son consistentes con los nuestros.

En nuestro estudio, menos de $15 \%$ de los pacientes sensibilizados al kiwi manifestaron síntomas después de haberlo comido; esta diferencia en las cantidades probablemente no es exclusiva de los alimentos, pues un estudio multicéntrico realizado en Europa mostró que un poco más de $80 \%$ de los pacientes sensibilizados a algún tipo de pasto tuvo síntomas relacionados con la exposición a él; en ese mismo estudio, en el resto de aeroalérgenos analizados, más de $60 \%$ de las sensibilizaciones registradas fueron clínicamente relevantes; ${ }^{10}$ así, nuestro estudio muestra que la prueba cutánea positiva al kiwi guarda poca relación con las molestias clínicas. Posiblemente, diferencias en la potencia alergénica de los alérgenos utilizados para detectar la sensibilización desempeñan un papel preponderante. ${ }^{10}$

En relación con las molestias producidas por el consumo del fruto del kiwi, estas consisten principalmente en síntomas orales, en menor medida, urticaria, compromiso respiratorio y cardiovascular. ${ }^{3}$ Nosotros documentamos un caso de síndrome de la alergia oral y otro caso con síntomas gastrointestinales y cutáneos. Por otro lado, ninguno de los dos pacientes con alergia al kiwi mostró síntomas asociados con productos derivados del látex (síndrome látex-fruta).

Entre las principales limitaciones de este estudio se debe destacar la escasez de casos de alergia al kiwi, por otro lado, la obtención retrolectiva de los datos, ambas circunstancias que factiblemente modifican las cifras de prevalencia.

En resumen, la prevalencia de sensibilización al kiwi en población adulta guarda similitudes con la informada alrededor del mundo; en consecuencia, su detección de manera regular, mediante 1 realización de pruebas cutáneas específicas, debería ser una práctica habitual en la consulta del alergólogo. Por otro lado, ante la sensibilización al kiwi, ahora se sugiere interrogar sobre la presencia de síntomas tras su ingesta.

\section{Referencias}

1. Plants of the World Online Portal. [Sitio web]. Actinidia chinensis var. deliciosa. England: Royal Botanic Gardens, Kewgscience. [Actualización 2017]. Disponible en: https://ndb.nal.usda.gov/ndb/foods/show/1 70332 ?fgcd=\&manu=\&lfacet=\&format=\&count=\&max=25\&offset=\&sort=default\&order=asc\&qlookup=ki wi + fruit $\& d s=\& q t=\& q p=\& q a=\& q n=\& q=\& i n g=$

2. USDA Branded Food Products Database. [Sitio web]. Full report (all nutrients): 45212448, kiwi fruit, UPC: 78056286032. Estados Unidos: [Consultado 2017 Mar 30]. [aprox. 1 página]. Disponible en: https://ndb.nal. usda.gov/ndb/foods/show/188761 fgcd=\&manu=\&lfacet=\&format=\&count=\&max=50\&offset=\&sort=default \&order=asc\&qlookup=kiwi+fruit\&ds $=\& q t=\& q p=\& q a=\& q n=\& q=\& i n g$ 
3. Hassan AK, Venkatesh YP. An overview of fruit allergy and the causative allergens. Eur Ann Allergy Clin Immunol. 2015;47(6):180-187.

4. Allergome. [Sitio web]. Act d [fruit]. Italia: Mari A; 2017. Disponible en: http://www.allergome.org/ script/dettaglio.php?id_molecule=1697

5. Van-Kampen V, De-Blay F, Folletti I, Kobierski P, Moscato G, Olivieri M, et al. EAACI position paper: Skin prick testing in the diagnosis of occupational type I allergies. Allergy. 2013;68(5):580-584. DOI: 10.1111/all.12120

6. Burney PG, Potts J, Kummeling I, Mills EN, Clausen M, Dubakiene R, et al. The prevalence and distribution of food sensitization in European adults. Allergy. 2014;69(3):365-371 DOI: 10.1111/ all.12341

7. Bedolla-Barajas M, Bedolla-Pulido TR, Camacho-Peña AS, Gonzaléz-García E, Morales-Romero J. Food hypersensitivity in Mexican adults at 18 to 50 years of age: A questionnaire survey. Allergy Asthma Immunol Res. 2014;6(6):506-511. DOI: 10.4168/aair.2014.6.6.5118

8. Puente-Fernández C, Maya-Hernández RL, Flores-Merino MV, Romero-Figueroa MS, BedollaBarajas M, Domínguez-García MV. Self-reported prevalence and risk factors associated with food hypersensitivity in Mexican young adults. Ann Allergy Asthma Immunol. 2016;116(6):523-527. DOI: 10.1016/j.anai.2016.03.01

9. Mahesh PA, Wong GW, Ogorodova L, Potts J, Leung TF, Fedorova O, et al. Prevalence of food sensitization and probable food allergy among adults in India: The EuroPrevall INCO Study. Allergy. 2016;71(7):1010-1019. DOI: 10.1111/all.12868

10. Burbach GJ, Heinzerling LM, Edenharter G, Bachert C, Bindslev-Jensen C, Bonini S, et al. GA(2) LEN skin test study II: Clinical relevance of inhalant allergen sensitizations in Europe. Allergy. 2009;64(10):1507-1515. DOI: 10.1111/j.1398-9995.2009.02089.x 